\title{
Heterologous Expression of Alteromonas macleodii and Thiocapsa roseopersicina [NiFe] Hydrogenases in Synechococcus elongatus
}

\author{
Philip D. Weyman ${ }^{1}$, Walter A. Vargas ${ }^{1 \alpha}$, Yingkai Tong ${ }^{1}$, Jianping $\mathrm{Yu}^{2}$, Pin-Ching Maness ${ }^{2}$, Hamilton 0. \\ Smith ${ }^{1}$, Qing $X^{1}{ }^{1 *}$
}

1 Department of Synthetic Biology and Bioenergy, The J. Craig Venter Institute, Rockville, Maryland, United States of America, 2 National Renewable Energy Laboratory, Golden, Colorado, United States of America

\begin{abstract}
Oxygen-tolerant [NiFe] hydrogenases may be used in future photobiological hydrogen production systems once the enzymes can be heterologously expressed in host organisms of interest. To achieve heterologous expression of [NiFe] hydrogenases in cyanobacteria, the two hydrogenase structural genes from Alteromonas macleodii Deep ecotype (AltDE), hynS and hynL, along with the surrounding genes in the gene operon of HynSL were cloned in a vector with an IPTGinducible promoter and introduced into Synechococcus elongatus PCC7942. The hydrogenase protein was expressed at the correct size upon induction with IPTG. The heterologously-expressed HynSL hydrogenase was active when tested by in vitro $\mathrm{H}_{2}$ evolution assay, indicating the correct assembly of the catalytic center in the cyanobacterial host. Using a similar expression system, the hydrogenase structural genes from Thiocapsa roseopersicina (hynSL) and the entire set of known accessory genes were transferred to S. elongatus. A protein of the correct size was expressed but had no activity. However, when the 11 accessory genes from AltDE were co-expressed with hynSL, the $T$. roseopersicina hydrogenase was found to be active by in vitro assay. This is the first report of active, heterologously-expressed [NiFe] hydrogenases in cyanobacteria.
\end{abstract}

Citation: Weyman PD, Vargas WA, Tong Y, Yu J, Maness P-C, et al. (2011) Heterologous Expression of Alteromonas macleodii and Thiocapsa roseopersicina [NiFe] Hydrogenases in Synechococcus elongatus. PLoS ONE 6(5): e20126. doi:10.1371/journal.pone.0020126

Editor: Francisco Rodriguez-Valera, Universidad Miguel Hernandez, Spain

Received February 23, 2011; Accepted April 13, 2011; Published May 26, 2011

Copyright: (C) 2011 Weyman et al. This is an open-access article distributed under the terms of the Creative Commons Attribution License, which permits unrestricted use, distribution, and reproduction in any medium, provided the original author and source are credited.

Funding: This work was supported by the U.S. Department of Energy, the Hydrogen, Fuel Cells, and Infrastructure Technology Program (DE-FG36-05GO15027). The funder had no role in study design, data collection and analysis, decision to publish, or preparation of the manuscript.

Competing Interests: The employer of the authors (J. Craig Venter Institute) is considering filing a patent based on research presented in this paper. However, this does not alter the authors' adherence to all the PLOS ONE policies on sharing data and materials.

*E-mail: qxu2019@gmail.com

a Current address: Centro Hispano-Luso de Investigaciones Agrarias (CIALE), Departamento de Microbiología y Genética, Universidad de Salamanca, Salamanca Spain

\section{Introduction}

Hydrogen $\left(\mathrm{H}_{2}\right)$ production from photosynthetic microorganisms is an attractive strategy to store solar energy as a fuel [1]. $\mathrm{H}_{2}$ fuel cells can provide carbon-free power; however, most $\mathrm{H}_{2}$ currently in use derives from fossil fuels [1]. Development of photobiological $\mathrm{H}_{2}$ production using photosynthetic microorganisms such as cyanobacteria and micro-algae can provide an alternative to fossil fuels by using the energy of the sun to convert $\mathrm{H}_{2} \mathrm{O}$ into $\mathrm{H}_{2}$.

$\mathrm{H}_{2}$ can be produced by cyanobacteria using either nitrogenase or hydrogenase enzymes [2]. Hydrogenases catalyze the reversible reduction of protons to $\mathrm{H}_{2}$ and can be divided into three phylogenetically-distinct categories that correlate with the metal composition of the active site: [FeFe], $[\mathrm{NiFe}]$, and the $[\mathrm{Fe}]$ hydrogenases of methanogens [3,4]. Two different groups of [NiFe] hydrogenases, the uptake hydrogenases and the bidirectional hydrogenases, have been found in many cyanobacterial genomes [2]. The uptake hydrogenases in cyanobacteria function largely in recycling $\mathrm{H}_{2}$ produced as a byproduct of nitrogen fixation while bidirectional hydrogenases have been implicated in disposing of excess reductant as $\mathrm{H}_{2}[5,6]$.

$\mathrm{H}_{2}$ production from photosynthetic microbes such as cyanobacteria requires hydrogenases to be tolerant of oxygen produced from photosynthesis if $\mathrm{H}_{2}$ production is to occur during daytime. Of the major categories of hydrogenases, $[\mathrm{FeFe}]$ hydrogenases are the most $\mathrm{O}_{2}$-sensitive and are irreversibly destroyed by exposure to oxygen [7]. Most [NiFe] hydrogenases are temporarily inactivated by $\mathrm{O}_{2}$ but can be reactivated upon returning to anaerobic conditions given sufficient reducing conditions [7]. All cyanobacterial $[\mathrm{NiFe}]$ hydrogenases studied thus far are sensitive to $\mathrm{O}_{2}$ and function only briefly in aerobic conditions before being inactivated [8]. Nonetheless, several [NiFe] hydrogenases from other microorganisms maintain activity in the presence of oxygen, including those from Ralstonia eutropha [9], Rubrivivax gelatinousous [10] and Alteromonas macleodii [11].

Using $\mathrm{O}_{2}$-tolerant hydrogenases in future cyanobacterial hydrogen production systems will require their heterologous expression in cyanobacteria, and expression is currently a barrier to the wide-spread use of foreign hydrogenases in cyanobacteria. The catalytic core of [NiFe] hydrogenases generally consists of two subunits, one large $(c a .60 \mathrm{kDa})$ and one small $(c a .30 \mathrm{kDa})$. The large subunit contains the $[\mathrm{NiFe}]$ catalytic site and requires an extensive set of accessory proteins to assemble an active catalytic site [12]. Maturation of the small subunit is not as well understood, but some $[\mathrm{NiFe}]$ hydrogenases require specific accessory proteins to assist in this process [13]. The accessory proteins are usually 
specific for the hydrogenase with which they have co-evolved, but may be active on closely related hydrogenases from another species $[14,15]$.

The complete and clustered set of accessory genes from AltDE may simplify the task of heterologous expression [16]; however, other organisms, including Thiocapsa roseopersicina that contains a related $[\mathrm{NiFe}]$ hydrogenase, have accessory genes distributed throughout the genome [17]. Although the T. roseopersicina genome has not been sequenced, mutational analysis has identified several accessory genes (hynD, hupCDHIK and hypC1C2DEF) [17,18,19]. Here, we report the heterologous expression of active [NiFe] hydrogenases from AltDE and T. roseopersicina in the cyanobacterium Synechococcus elongatus PCG7942. Heterologous expression of an active $[\mathrm{NiFe}]$ hydrogenase has not been reported previously in cyanobacteria, and the development of systems for heterologous expression of hydrogenases in cyanobacteria may open up new possibilities for photobiological hydrogen production.

\section{Results}

\section{Construction of the hoxYH hydrogenase mutant in}

\section{S. elongatus PCC7942}

The genome of S. elongatus PCG7942 contains the hoxrH genes encoding one [NiFe] bidirectional hydrogenase (HoxYH) (http:// genome.jgi-psf.org/synel/synel.home.html). To eliminate background hydrogenase activity in $S$. elongatus to better detect the activity from heterologously-expressed enzymes, we knocked out the endogenous hydrogenase by transforming with a plasmid (pPW416) that would replace the hox $Y H$ genes with an antibiotic cassette via DNA recombination. Complete segregation of the mutation-containing sequence in $S$. elongatus was confirmed by PCR and southern blot analysis (Figs. S1 and S2). The S. elongatus knockout mutant, named PW416, lacked any detectable hydrogenase activity (Table S2).

\section{Expression of AltDE HynSL hydrogenase in S. elongatus PCC7942}

AltDE contains one [NiFe] hydrogenase, HynSL, which has been characterized as oxygen-tolerant and thermostable [11]. The structural genes, hynSL, are surrounded by 11 genes encoding accessory proteins involved in the assembly and maturation of the [NiFe] hydrogenase catalytic site and the hydrogenase complex. A subset of these genes has been determined to play a critical role in hydrogenase maturation [16]. In order to express HynSL in $S$. elongatus, plasmid $\mathrm{pRC4} 1$ was constructed by cloning the hydrogenase structural genes, hynSL, and the 11 adjacent genes into an expression plasmid [16] (Fig. 1). This plasmid contains a copy of the lacI gene [20] and allows for expression of the AltDE hydrogenase operon from the IPTG-inducible $\mathrm{P}_{\text {Trc }}$ promoter. The plasmid pRC41 also contains flanking sequence for insertion of the hydrogenase gene cluster into "neutral site I" (NSI) of the $S$. elongatus chromosome via homologous DNA recombination [21]. The construct was introduced into the S. elongatus hydrogenase knockout strain, PW416, to create strain RC41.

To examine the expression of HynSL, RC41 cultures expressing the AltDE hydrogenase gene operon were grown and induced with IPTG, and protein extracts from lysed cells were separated on an SDS-PAGE gel for immunoblotting. Western blotting was performed with antiserum raised against the $T$. roseopersicina $[\mathrm{NiFe}]$ hydrogenase large subunit, HynL. When induced with IPTG, a single band corresponding to the mature form of the AltDE HynL $(67 \mathrm{kDa})$ was detected (Fig. 2A). Without IPTG induction, no HynL band was detected. As a control, a duplicate gel was stained with Coomassie blue to confirm equal loading in each lane (Fig. 2B). IPTG was added to a final concentration of 5, 20, 100, or $200 \mu \mathrm{M}$ to RC41 cultures to determine the optimal concentration of IPTG for protein expression. Western blotting analysis indicated that $100 \mu \mathrm{M}$ IPTG yielded maximal expression of HynL (data not shown) and this concentration of IPTG was used for all future experiments.

To determine whether the AltDE HynSL protein was expressed with an active catalytic site in $S$. elongatus PCC7942, in vitro $\mathrm{H}_{2}$ evolution assays were performed with protein extracts from RC41. As expected, no hydrogenase activity was detected in the PW416 $(\Delta$ hox $Y H)$ strain (Fig. 2C). Hydrogenase activity was detected in strain RC41 expressing both hydrogenase and accessory proteins from AltDE, and activity was strongly induced by IPTG. The activity from the heterologously expressed hydrogenase in RC41 represented one tenth of the native activity in the wild type $S$. elongatus strain (Fig. 2G). A small amount of activity was detected in the absence of IPTG induction, indicating slightly leaky expression from the $\mathrm{P}_{\text {Trc }}$ promoter.

\section{Expression of $T$. roseopersicina HynSL hydrogenase in \\ S. elongatus PCC7942}

In addition to expressing AltDE HynSL, we expressed its related stable hydrogenase, HynSL, from T. roseopersicina in S. elongatus. We assembled the sequences of $T$. roseopersicina accessory genes into two sets of plasmids. In the first set of plasmids, pHyn4-NSII, hynD (encoding the protease) as well as hupK and hypC1C2DEF were assembled along with the hydrogenase structural genes hynSL in an IPTG-inducible vector (Fig. 3), which could integrate into the

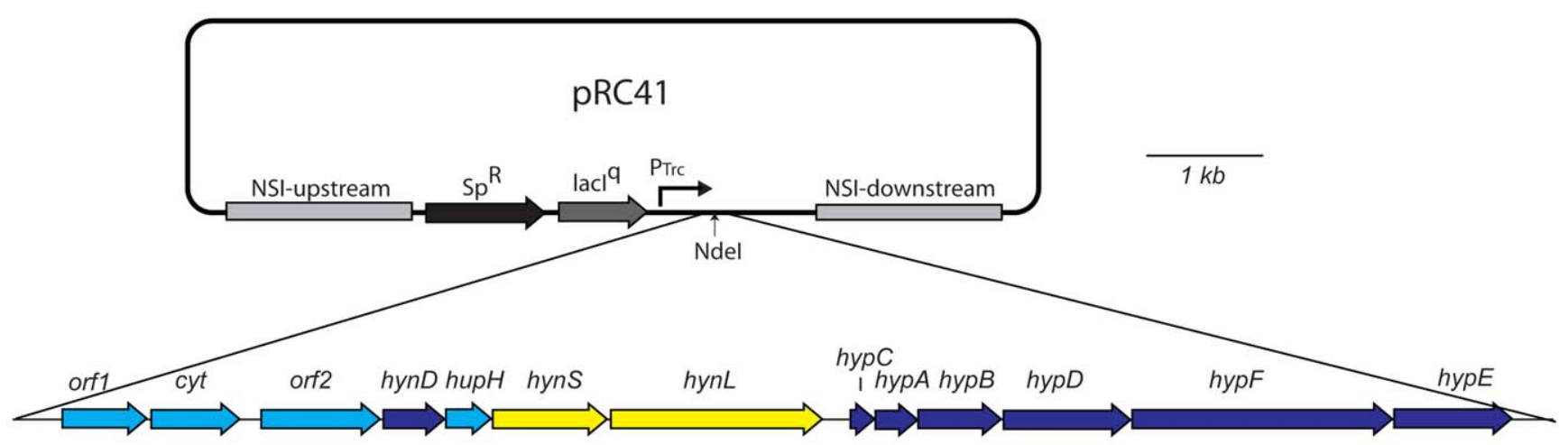

Figure 1. Diagram of pRC41 used for expression of AltDE hydrogenase HynSL in S. elongatus. Arrows indicate the direction of open reading frames. The figure was drawn to scale.

doi:10.1371/journal.pone.0020126.g001 


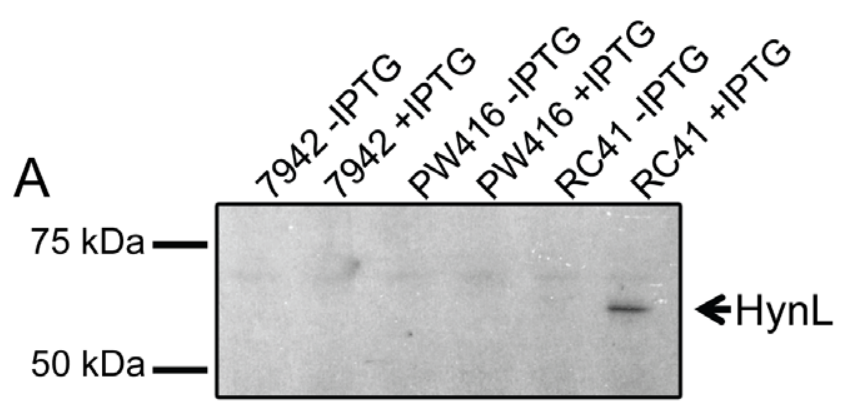

B
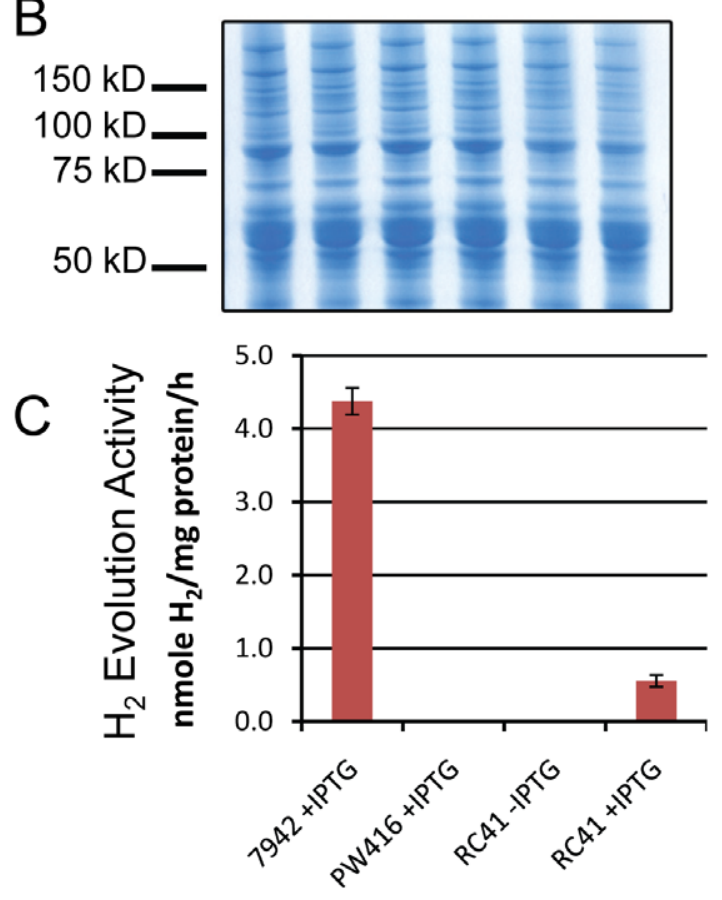

Figure 2. Heterologous expression of AltDE hydrogenase HynSL in S. elongatus. A. Western blotting of protein extracts from S. elongatus cell cultures. Each lane contains $25 \mu \mathrm{g}$ total proteins from cells treated with or without IPTG. The arrow indicates the position of the HynL band. B. Coomassie blue staining of a duplicate protein gel as in Panel A showing equal loading in each lane. C. Determination of the hydrogenase activity of the heterologously expressed HynSL. In vitro hydrogen evolution assays were performed on protein samples described in Panel A. Two controls, 7942-IPTG and PW416-IPTG, were not included since their activity levels are same as those from 7942+IPTG and PW416+IPTG, respectively. Error bars indicate standard deviations of the mean from at least three samples. The experiment was repeated at least three times with similar results.

doi:10.1371/journal.pone.0020126.g002

"neutral site II" (NSII) of S. elongatus through homologous recombination [21]. The plasmid was introduced into the hydrogenase knockout mutant (PW416) to make strain Hyn4. We further modified plasmid pHyn4-NSII to include two additional genes, isp1 and isp2. These two genes are found between hynS and hynL in the T. roseopersicina chromosome [22]. They were removed in the construction of pHyn4-NSII but were added back downstream of the hypDEF genes in the construction of pHyn5-NSII (Fig. 3). Isp1 and Isp2 are not predicted to play a role in hydrogenase maturation but rather confer electron transfer necessary for in vivo hydrogenase activity [23]. Plasmid pHyn5 was mobilized into the hydrogenase knockout strain to make Hyn5.

In the second set of plasmids, pHup-NSI, the T. roseopersicina accessory genes hupCDHI and an ORF of unknown function were assembled in an IPTG-inducible vector, which could recombine at the $S$. elongatus NSI locus (Fig. 3). After mobilization of pHup-NSI into the hydrogenase knockout mutant, PW416, the resulting strain was named Hup. The pHup-NSI plasmid was also mobilized into Hyn4 and Hyn5 to create strains Hyn4/Hup and Hyn5/Hup, respectively.

S. elongatus cell cultures expressing different combinations of $T$. roseopersicina structural and accessory genes were induced with IPTG, and protein extracts prepared from lysed cells were analyzed by immunoblot after SDS-PAGE electrophoresis using anti-HynL antisera. A band corresponding to the correct size of the mature form of HynL was detected $(62 \mathrm{kDa})$, and increased expression was observed in the presence of IPTG (Fig. 4). Similar levels of HynL expression were observed for all strains that contained HynSL (Fig. 4). In vitro hydrogen evolution assays were performed to determine whether the expressed hydrogenase possessed activity. No activity was detected in strains Hyn4, Hyn5, Hyn4/Hup or Hyn5/Hup (data not shown).

\section{Expression of the T. roseopersicina HoxYH in S. elongatus PCC7942}

Since the T. roseopersicina HynSL hydrogenase was not properly assembled into its active form in $S$. elongatus using the native cyanobacterial accessory proteins, we sought to determine whether an enzyme with greater similarity to the cyanobacterial hydrogenase such as HoxYH might be successfully assembled and possess activity when heterologously expressed. T. roseopersicina also contains a bidirectional hydrogenase encoded by genes hox $Y H$, which has been shown to interact with the diaphorase subunit encoded by genes hoxEFU [23]. The small and large subunits are 44 and 54 percent similar, respectively, to the native bidirectional hydrogenase in $S$. elongatus [24]. Given the similarities between the two proteins, a functional T. roseopersicina HoxYH may be able to be assembled and processed by the native $S$. elongatus accessory proteins. To express the $T$. roseopersicina HoxYH hydrogenase in S. elongatus, the hoxEFUYH gene cluster was cloned upstream of the hupCDHIorf genes, resulting in plasmid pHoxhup-NSI (Fig. 3). pHoxhup-NSI with the combined gene cluster was transformed into S. elongatus PW416 and Hyn5, to make strains Hoxhup and Hyn5/Hoxhup, respectively. The $S$. elongatus cultures were induced with IPTG, and protein extracts from lysed cells were used for immunoblot analysis. A $62 \mathrm{kDa}$ protein band corresponding to the correct size of the mature form of HynL was observed (Fig. 4). in vitro hydrogen evolution assay was performed to determine if an active hydrogenase was produced. No activity was detected for Hoxhup or Hyn5/ Hoxhup that contains additional T. roseopersicina accessory genes hynDhupKhypC1C2DEF (data not shown). Thus, maturation of a functional HoxYH from $T$. roseopersicina requires additional accessory genes that are not able to be complemented by genes in the cyanobacterial host.

\section{Co-expression of $T$. roseopersicina HynSL and AltDE accessory proteins in S. elongatus PCC7942}

Since HynSL from AltDE was active when expressed in $S$. elongatus RC41, we tried to co-express the $T$. roseopersicina HynSL hydrogenase with accessory proteins from AltDE. Plasmid pWAV10 (Fig. 3) was constructed to carry only the 11 genes encoding AltDE accessory proteins and was introduced into the NSI locus of $S$. elongatus strains Hyn4 and Hyn5 described above, creating Hyn4/Wav10 and Hyn5/Wav10, respectively. To serve as a control, pWAV10 was also introduced into the $S$. elongatus hydrogenase knockout strain, PW416, 


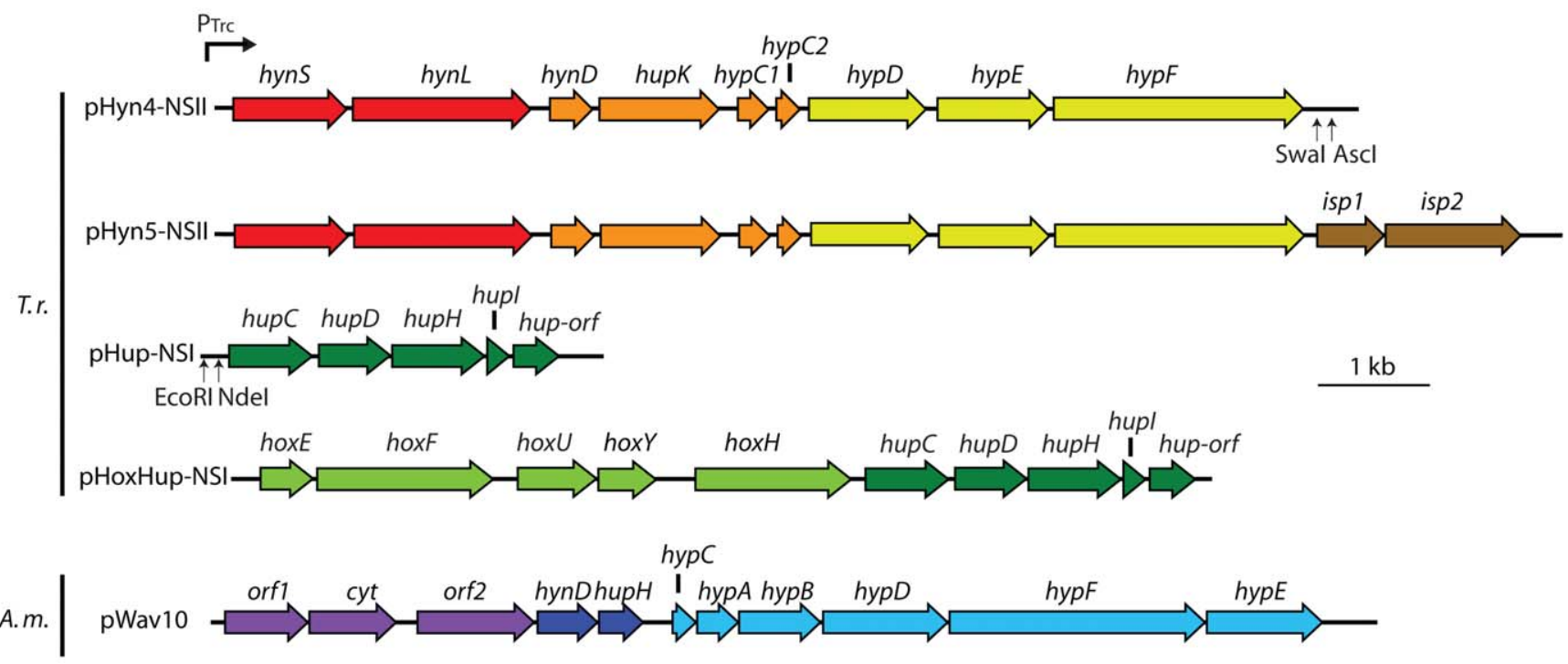

Figure 3. Diagram of constructs used in heterologous expression of T. roseopersicina HynSL in S. elongatus. Arrows indicate the direction of open reading frames. The figure was drawn to scale. doi:10.1371/journal.pone.0020126.g003

creating Wav10 that contained all AltDE accessory genes, but no structural genes. Expression of the HynL protein in these strains was verified by SDS-PAGE and Western blotting (Fig. 5A). A duplicate gel was stained with Coomassie blue to confirm equal loading in each lane as a loading control (Fig. 5B). In vitro $\mathrm{H}_{2}$ evolution assays were performed to determine whether the heterologously expressed T. roseopersicina HynSL hydrogenase was active when expressed in the cyanobacterium with the AltDE accessory proteins. Hydrogen evolution activity was detected in both Hyn4/Wav10 and Hyn5/Wav10 strains at similar levels in both strains (Fig. 5C). Activity from $\mathcal{T}$. roseopersicina HynSL was approximately one tenth of the activity of AltDE HynSL (Figs. 2C and 5C). To determine if the AltDE accessory proteins were capable of producing an active $T$. roseopersicina $\mathrm{Hox} \mathrm{YH}$, the plasmid pWav10 was introduced into the S. elongatus strain Hoxhup. The resulting strain, Hoxhup/ Wav10, did not produce any $\mathrm{H}_{2}$ during in vitro hydrogen evolution assays after IPTG induction (Fig. 5C).

\section{Discussion}

We have expressed the $[\mathrm{NiFe}]$ hydrogenases from AltDE and $T$. roseopersicina in the heterologous host, S. elongatus PCG7942. [NiFe] hydrogenases have been previously expressed in heterologous bacterial hosts $[14,15,25,26,27,28]$, but to our knowledge this is the first $[\mathrm{NiFe}]$ hydrogenase to be heterologously expressed in a cyanobacterium with a fully assembled active site. The ability to heterologously express properly assembled [NiFe] hydrogenases in cyanobacteria has been a technical barrier hindering widespread biotechnological application of hydrogenases with unique properties. In direct photobiological hydrogen production systems, electrons derived from water oxidation through oxygenic photosynthesis are used directly for hydrogen production without being stored as a fixed-carbon intermediate [29]. Development of such a system requires either low partial pressures of $\mathrm{O}_{2}$ as has been achieved for brief periods with eukaryotic green algae [30], or the use of an $\mathrm{O}_{2}$-tolerant hydrogenase. We have taken a step toward

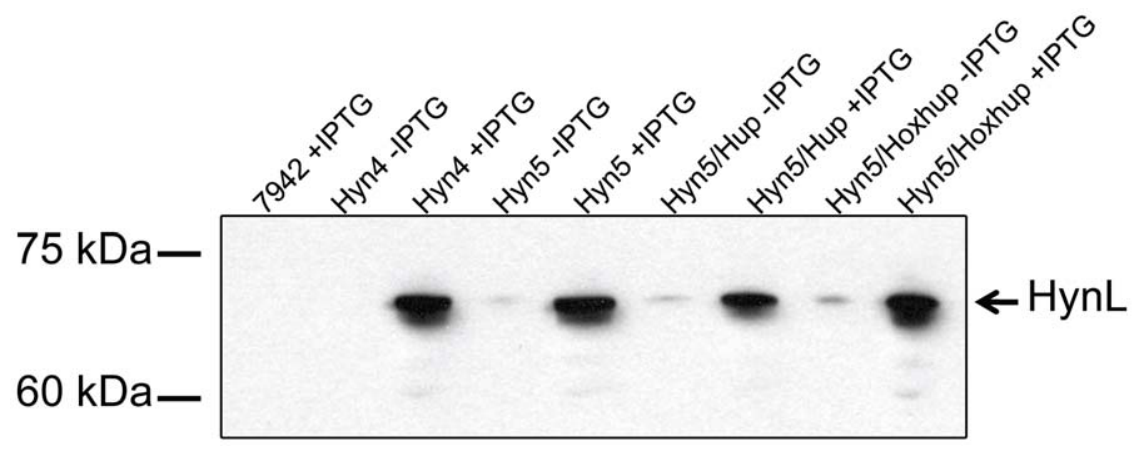

Figure 4. Detection of the $T$. roseopersicina hydrogenase large subunit $\mathbf{H y n L}$ in S. elongatus. Western blotting was performed on protein extracts from S. elongatus strains (wild-type PCC7942, Hyn4, Hyn5, Hyn5/Hup, and Hyn5/Hoxhup) by using anti-HynL antisera. Hyn4/Hup was not included since the expression level in this strain was similar to that in Hyn4. Each lane contains $25 \mu \mathrm{g}$ total proteins from cells treated with or without IPTG. Strain Hyn4 carries the T. roseopersicina genes hynSL, hynD, hupK, and $h y p C_{1} C_{2} D E F$ while strain Hyn5 carries the genes from Hyn4 as well as isp 1 and isp2. Strain Hyn5/Hup contains the T. roseopersicina genes hupCDHlorf and the genes from Hyn5. Strain Hyn5/Hoxhup contains the $T$. roseopersicina genes hoxEFUYH in addition to the genes from Hyn5/Hup.

doi:10.1371/journal.pone.0020126.g004 
A

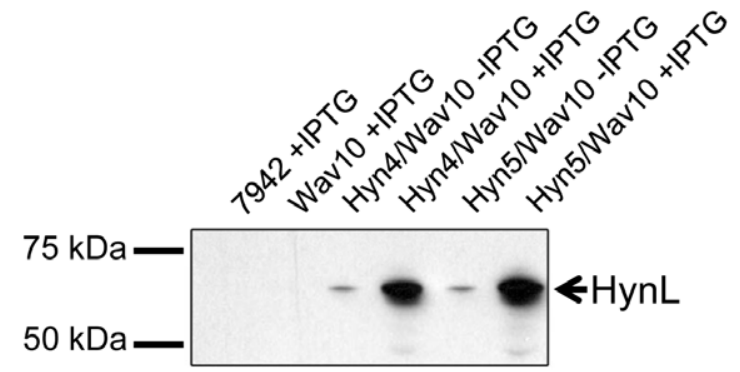

B

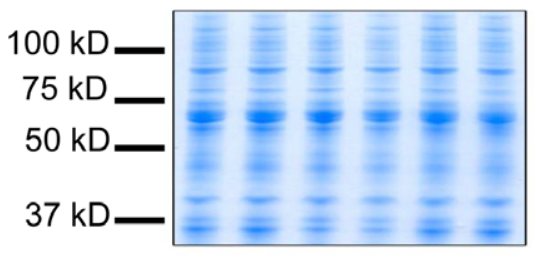

C

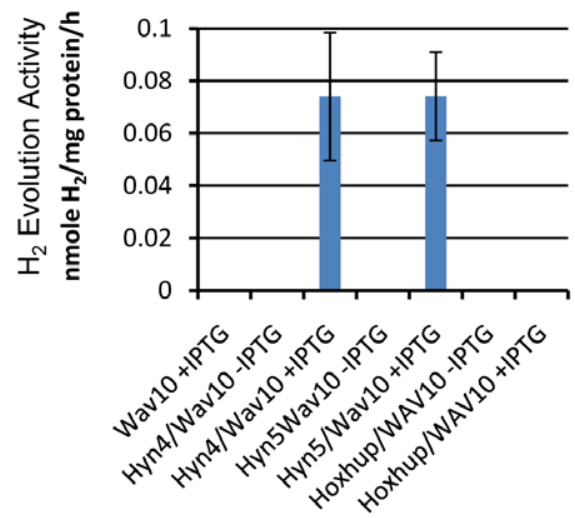

Figure 5. Expression of $T$. roseopersicina hydrogenase with accessory proteins from AltDE in S. elongatus. A. Detection of the $T$. roseopersicina hydrogenase large subunit HynL in S. elongatus. Western blotting was performed with protein extracts of $S$. elongatus strains, wild-type PCC7942, Wav10, Hyn4/Wav10, and Hyn5/Wav10. Each lane contains $25 \mu \mathrm{g}$ total proteins from samples treated with or without IPTG. B. Coomassie blue staining of a duplicate protein gel as in panel A showing equal loading in each lane. C. Determination of the hydrogenase activity of the heterologously expressed HynSL. In vitro hydrogen evolution assays were performed on protein samples collected from S. elongatus strains Wav10, Hyn4/Wav10, Hyn5/Wav10, and HoxHup/Wav10 after treatment with or without IPTG induction. Wav10-IPTG was not included since the activity in this negative control was not affected by IPTG induction. Error bars indicate standard deviations of the mean from at least three samples. The experiment was repeated at least three times with similar results.

doi:10.1371/journal.pone.0020126.g005

the latter approach by expressing a functional $\mathrm{O}_{2}$-tolerant $[\mathrm{NiFe}]$ hydrogenase in cyanobacteria. While the AltDE hydrogenase we used is only partially tolerant of up to $1-3$ percent $\mathrm{O}_{2}$, their expression will enable further progress on photobiological hydrogen production using the many genetic tools available for cyanobacteria [31].

The set of AltDE accessory proteins that was found to be required for maximal activity of AltDE HynSL when heterologously expressed in Escherichia coli included HypCABDFE, the protease $\mathrm{HynD}, \mathrm{HupH}$, and a protein of unknown function, Orf2 [16]. The S. elongatus strain RC41 expressed the above set of accessory proteins along with Orfl and Cyt. The AltDE proteins Orfl and Cyt were not found to be necessary for maximal activity when HynSL was heterologously expressed in E. coli, but it is unknown whether these proteins affect the maturation efficiency of HynSL in S. elongatus PCC7942. The set of AltDE accessory proteins contained in Wav10 was the same as those found on RC41 and was also sufficient to allow for expression of a functional T. roseopersicina hydrogenase HynSL in S. elongatus. When expressed in $S$. elongatus, AltDE HynSL activity was higher than $T$. roseopersicina HynSL activity, and this may reflect lower levels of activity of the AltDE accessory proteins when acting on the $T$. roseopersicina HynSL. Similarly, lower levels of activity were detected when HynSL was expressed with the AltDE accessory proteins in E. coli compared to expression of AltDE HynSL [16].

We assembled the genes encoding the entire known set of $T$. roseopersicina accessory proteins into an artificial operon driven by the $\mathrm{P}_{\text {Trc }}$ promoter. This set of accessory genes was not sufficient to produce an active HynSL hydrogenase. The $\mathcal{T}$. roseopersicina operon included many genes that were also included in the AltDE cluster such as hypCDEF, hynD, and hupH. HupH from AltDE was required for maximal assembly and maturation of AltDE HynSL in E. coli [16] and was also identified in T. roseopersicina; however, the HupH sequences from AltDE and T. roseopersicina share only $11 \%$ similarity. It is unknown whether the two HupH proteins function similarly in their respective hosts. Absent from the group of known $T$. roseopersicina accessory genes is hyp $A B$. These genes encode proteins that belong to the set of accessory proteins (HypABCDEF) that is found in all species containing [NiFe] hydrogenases [12]. HypAB function to add the $\mathrm{Ni}$ atom to the nascent $[\mathrm{NiFe}]$ catalytic site. In some systems, the absence of HypAB has been complemented by high concentrations of nickel, but these genes are required for maximal hydrogenase activity [32]. When the AltDE hypAB genes were co-expressed with in $T$. roseopersicina hynSL, hynD, hypC1C2DEF in E. coli, no hydrogenase activity was detected [16]. This suggests that the AltDE HypAB proteins alone cannot effectively interact with the rest of the $\mathcal{T}$. roseopersicina accessory proteins and that the additional proteins (HupH or Orf2) encoded by Wavl0 are important to the maturation of the T. roseopersicina hydrogenase.

We also attempted to express the bidirectional hydrogenase from T. roseopersicina, HoxYH, in S. elongatus. Even after co-expression with the entire set of known $T$. roseopersicina accessory proteins or with the accessory proteins from AltDE, no hydrogenase activity could be detected from strains expressing HoxYH. Missing from the accessory genes known to contribute to maturation of HoxYH is the endo-peptidase, HoxW. This peptidase activity is apparently not able to be complemented by the native $S$. elongatus HoxW that was purposely left intact when constructing the hox $Y H$ mutant strain (PW416). This finding is consistent with a previous report demonstrating that HoxW cannot be complemented by other proteases for processing T. roseopersicina HoxYH [33].

Heterologous expression of $\mathrm{NiFe}$ hydrogenases with fully assembled active sites in cyanobacteria will enable further study of hydrogenase expression in photosynthetic prokaryotic hosts such as cyanobacteria. Further studies are in need to increase expression, improve oxygen tolerance to the atmospheric levels, and establish coupling of the heterologous hydrogenase to the photosynthetic electron transport chain. The successful outcomes will lead to cyanobacterial strains capable of producing hydrogen simultaneously during photosynthesis.

\section{Materials and Methods}

\section{Strains and growth conditions}

Molecular biology techniques were performed according to [34]. E. coli cultures were grown at $30^{\circ} \mathrm{C}$ or $37^{\circ} \mathrm{C}$ in Luria- 
Bertaini (LB) broth, or on LB agar plates supplemented with antibiotics as needed (spectinomycin, $50 \mu \mathrm{g} \mathrm{ml}^{-1}$, kanamycin, $25 \mu \mathrm{g} \mathrm{ml}^{-1}$, chloramphenicol, $25 \mu \mathrm{g} \mathrm{ml}^{-1}$ ). Cyanobacteria were grown in BG11 liquid media [35] or on BG11 agar plates supplemented with antibiotics as needed (spectinomycin, $10 \mu \mathrm{g} \mathrm{ml}^{-1}$; kanamycin, $10 \mu \mathrm{g} \mathrm{ml}^{-1}$; erythromycin, $5 \mu \mathrm{g} \mathrm{ml}^{-1}$ ). Cells were grown under continuous illumination $\left(40 \mu \mathrm{E} \mathrm{m}^{-2} \mathrm{~s}^{-1}\right)$ at $28^{\circ} \mathrm{C}$ in $100 \mathrm{ml}$ cultures with constant shaking or in $500 \mathrm{ml}$ cultures with constant stirring and aeration. Cultures were induced with Isopropyl $\beta$-D-1-thiogalactopyranoside (IPTG) at a final concentration of $100 \mu \mathrm{M}$ and $\mathrm{NiCl}_{2}$ at a final concentration of $0.5 \mu \mathrm{M}$ for 24 hours before the cultures were used for experiments. Cultures of AltDE and T. roseopersicina were grown as previously described [11].

\section{Plasmid construction and genetic manipulation of cyanobacteria}

Plasmid pPW416 was constructed to knockout the hydrogenase structural genes hoxYH in S. elongatus PCC7942 [36]. This vector was designed to leave the upstream hox $U$ and downstream hoxW intact (Table 1). To make pPW416, a four piece ligation was performed using the following DNA pieces: 1) A PCR product containing resistance genes to erythromycin $\left(\mathrm{Em}^{\mathrm{R}}\right)$ and chloramphenicol $\left(\mathrm{Cm}^{\mathrm{R}}\right)$ amplified from pRL2948a using primers EmCm-F and EmCm-R (Table 2) and digested with XhoI and SpeI, 2) A PCR product containing 1-kb of sequence upstream of $S$. elongatus hox $Y$ amplified using primers Hox 11 and Hox 12 and digested with HindIII and XhoI, 3) a PCR product containing $1-\mathrm{kb}$ of sequence downstream of hox $H$ amplified with

Table 1. Bacterial strains and plasmids used in this study.

\begin{tabular}{|c|c|c|}
\hline \multicolumn{3}{|l|}{ Escherichia coli strains } \\
\hline Strain name & Features & Reference \\
\hline $\mathrm{DH} 10 \mathrm{~B}$ & $\begin{array}{l}\mathrm{F}-\text { mcrA } \Delta(\text { mrr-hsdRMS-mcrBC) } \varphi 80 / a c Z \Delta M 15 \Delta / a c X 74 \text { rec } \\
\mathrm{A} 1 \text { endA1 araD139 } \Delta(\text { ara, leu }) 7697 \text { galU galK } \lambda \text { - rpsL nupG }\end{array}$ & Invitrogen \\
\hline $\begin{array}{l}\text { Alteromonas macleodii "deep } \\
\text { ecotype" DSMZ } 17117\end{array}$ & Wild type & {$[38]$} \\
\hline Thiocapsa roseopersicina BBS & Wild type & {$[39]$} \\
\hline \multicolumn{3}{|l|}{ Synechococcus elongatus strains } \\
\hline S. elongatus PCC 7942 & Wild type & Pasteur Culture Collection \\
\hline PW416 & PCC 7942, $\Delta h o x Y H:: \mathrm{Em}^{\mathrm{R}}$ & This work \\
\hline RC41 & AltDE hydrogenase operon inserted into NSI site [21] of strain PW416, $\mathrm{Em}^{\mathrm{R}}, \mathrm{Sp}^{\mathrm{R}}$ & This work \\
\hline Hyn4 & $\begin{array}{l}\text { hynShynLhynDhupKhypC1hypC2hypDhypEhypF from T. } \\
\text { roseopersicina in NSII site [21] of strain PW } 416, \mathrm{Em}^{R}, \mathrm{Km}^{\mathrm{R}}\end{array}$ & This work \\
\hline Hyn5 & $\begin{array}{l}\text { hynShynLhynDhupKhypC1hypC2hypDhypEhypFisp1isp2 from T. } \\
\text { roseopersicina in NSIl site [21], of strain PW416, } \mathrm{Km}^{\mathrm{R}}, \mathrm{Em}^{\mathrm{R}}\end{array}$ & This work \\
\hline Hup & hupCDHlorf from T. roseopersicina in NSI site of strain PW416, $\mathrm{Sp}^{\mathrm{R}}, \mathrm{Em}^{\mathrm{R}}$ & This work \\
\hline HoxHup & hoxEFUYHhupCDHlorf from T. roseopersicina in NSI site of strain PW416, $\mathrm{Sp}^{\mathrm{R}}, \mathrm{Em}^{\mathrm{R}}$ & This work \\
\hline Wav10 & $\begin{array}{l}\text { orf1/cyt/orf2/hynD/hupH/hypC/hypA/hypB/hypD/hypF/hypE from } \\
\text { AltDE in NSI site of strain PW416, } \mathrm{Sp}^{\mathrm{R}}, \mathrm{Km}^{\mathrm{R}}, \mathrm{Em}^{\mathrm{R}}\end{array}$ & This work \\
\hline Hyn4/Wav10 & pWav10 construct in NSI site, pHyn4 construct in NSIl site of strain PW416, $\mathrm{Sp}^{\mathrm{R}}, \mathrm{Km}^{\mathrm{R}}, \mathrm{Em}^{\mathrm{R}}$ & This work \\
\hline Hyn5/Wav10 & pWav10 construct in NSI site, pHyn5 construct in NSII site of strain PW416, $\mathrm{Sp}^{\mathrm{R}}, \mathrm{Km}^{\mathrm{R}}, \mathrm{Em}^{\mathrm{R}}$ & This work \\
\hline Hyn4/Hup & pHup construct in NSI site, pHyn4 construct in NSII site of strain PW416, $\mathrm{Sp}^{\mathrm{R}}, \mathrm{Km}^{\mathrm{R}}, \mathrm{Em}^{\mathrm{R}}$ & This work \\
\hline Hyn5/Hup & pHup construct in NSI site, pHyn5 construct in NSII site of strain PW416, $\mathrm{Sp}^{\mathrm{R}}, \mathrm{Km}^{\mathrm{R}}, \mathrm{Em}^{\mathrm{R}}$ & This work \\
\hline Hyn5/HoxHup & pHoxHup construct in NSI site, pHyn5 construct in NSII site of strain PW416, $\mathrm{Sp}^{\mathrm{R}}, \mathrm{Km}^{\mathrm{R}}, \mathrm{Em}^{\mathrm{R}}$ & This work \\
\hline Wav10/HoxHup & pWav10 construct in NSI site, pHoxHup-NSII construct in NSII site of strain PW416, $\mathrm{Sp}^{\mathrm{R}}, \mathrm{Km}^{\mathrm{R}}, \mathrm{Em}^{\mathrm{R}}$ & This work \\
\hline \multicolumn{3}{|l|}{ Plasmids: } \\
\hline pRL2948a & mob, oriT, sacB, $\mathrm{Em}^{\mathrm{R}} \mathrm{Cm}^{\mathrm{R}}$ & C. P. Wolk, unpublished data \\
\hline pTRC-NSI & Cloning vector, $\mathrm{Sp}^{\mathrm{R}}$ & {$[20]$} \\
\hline pTRC-NSII & Cloning vector, $\mathrm{Km}^{\mathrm{R}}$ & {$[20]$} \\
\hline pHoxHup-NSI & T.roseopersicina hoxEFUYH, hupCDHlorf cloned into pTRC-NSI & This work \\
\hline pHoxHup-NSII & T.roseopersicina hoxEFUYH, hupCDHlorf cloned into pTRC-NSII & This work \\
\hline pHup-NSI & T.roseopersicina hoxEFUYH, hupCDHlorf genes cloned into pTRC-NSI & This work \\
\hline pHyn4-NSII & T.roseopersicina hynSL, hynD, hupK, hypC1, hypC2, hypDEF genes cloned into pTRC-NSII & This work \\
\hline pHyn5-NSII & T.roseopersicina hynSL, hynD, hupK, hypC1, hypC2, hypDEF, isp 1, isp2 genes cloned into pTRC-NSII & This work \\
\hline pWav10 & $\begin{array}{l}\text { orf1/cyt/orf2/hynD/hupH/hypC/hypA/hypB/hypD/hypF/hypE inserted } \\
\text { into NSI site, } \Delta \text { hynSL, cloned into pTRC-NSI, Sp }{ }^{R}\end{array}$ & [16] \\
\hline pPW416 & S. elongatus hox region $\left(\Delta h o x Y H:: \mathrm{Em}^{\mathrm{R}}\right)$ cloned in pUC19, $\mathrm{Em}^{\mathrm{R}}, \mathrm{Cm}^{\mathrm{R}}$ & This work \\
\hline pRC41 & $\begin{array}{l}\text { orf1/cyt/orf2/hynD/hupH/hynS/hynL/hypC/hypA/hypB/hypD/hypF/hypE } \\
\text { inserted into NSI site, cloned into pTRC-NSI, Sp }{ }^{\mathrm{R}}\end{array}$ & {$[16]$} \\
\hline
\end{tabular}


Table 2. PCR primers used in this study.

\begin{tabular}{|c|c|}
\hline Primer Name & Sequence $\left(5^{\prime}-3^{\prime}\right)$ \\
\hline Isp-F & TATCAATTTAAATTGGCCAAGAAGCAGACCAAG \\
\hline Isp-R & GAGGCGCGCCTCATGTCAGTTCTTCTCCAC \\
\hline HupCDHlorf-F & TTAATCTCATATGCGGACCTGGCGGGGA \\
\hline HupCDHlorf-R & TCACTAGTGAGAGAAATTCCACTCCGG \\
\hline TrHox-F1 & $\begin{array}{l}\text { ACAGACCATGGAATTCGAGCTCAAGGAGGAATAA } \\
\text { CATATGAGTCTGCAGCAAGCCAAGCC }\end{array}$ \\
\hline TrHox-R1 & $\begin{array}{l}\text { AATAATCACCTGAAACGCGTCCCCGCCAGGTCCGCA } \\
\text { TATGTTATCCTCCCTTCAGCCGCGCCTGAGTGTGTCG }\end{array}$ \\
\hline $\mathrm{EmCm}-\mathrm{F}$ & TTATACTCGAGCACGTTCCATGGCCTCCAT \\
\hline $\mathrm{EmCm}-\mathrm{R}$ & AATATACTAGTCTGTCATGCCATCCGTAAGATGC \\
\hline Hox11 & AATCGAAGCTTCGAGTCCATAGCGATGGC \\
\hline Hox12 & TTATTGGATCCAACAGGACTGAAGACGACCTC \\
\hline Hox15 & TTAATCTCGAGATTGCGGAGATGGTTGAAGAC \\
\hline Hox16 & TAATTTCTAGAAATTGAGCAGGCACTGACTC \\
\hline
\end{tabular}

primers Hox 15 and Hox 16 and digested with SpeI and XbaI, and 4) pUC19 digested with HindIII and XbaI. After transformation into $E$. coli, the resulting plasmid was subsequently digested with XbaI and ligated with an XbaI-digested DNA fragment from pRL448 containing a kanamycin resistance gene $\left(\mathrm{Km}^{\mathrm{R}}\right)$. The resulting plasmid, pPW416, was confirmed by restriction digest and DNA sequencing. The plasmid was then transformed into S. elongatus as previously described [31], and the resulting strain was called PW416. Double DNA recombination was verified by sensitivity to kanamycin, and the strain was segregated by streaking cells on progressively increasing concentrations of erythromycin. The absence of the hox $Y H$ genes in the segregated PW416 strain was verified by PCR and southern hybridization.

The plasmid pHyn4 was previously described [16]. To make pHyn4-NSII, the entire cluster of T. roseopersicina genes in pHyn4 was digested with NdeI and AscI and ligated into a similarly digested pTRC-NSII. To make pHyn5-NSII, the isp1isp2 genes were amplified by PCR from $T$. roseopersicina genomic DNA using primers Isp-F and Isp-R, digested with SwaI and AscI, and ligated into SwaI and AscI-digested pHyn4-NSII. The resulting plasmid, pHyn5-NSII, contains the isp1isp2 genes downstream of the cluster including the hynSL, hynD, hupK, and hypC1C2DEF genes.

To make pHup-NSI, the genes encoding hupCDHI and an additional open reading frame (orf) of unknown function that is located after hupI [18] were amplified by PCR from T. roseopersicina genomic DNA using primers HupCDHIorf-F and HupCDHIorf$\mathrm{R}$. The resulting PCR product was digested with NdeI and SpeI and ligated into an NdeI/SpeI-digested pTRC-NSI. To make pHoxHup-NSI, the hoxEFUYH [17] genes were amplified from $T$. roseopersicina genomic DNA using primers TrHox-F1 and TrHox$\mathrm{R} 1$. These primers were designed so that each end of the resulting product contained 40-bp of sequence homology to the pHup-NSI vector. The plasmid, pHup-NSI was digested with EcoRI and NdeI and assembled with the hoxEFUYH PCR product using the one-step isothermal "chewback and anneal" (CBA) assembly method [37]. The resulting plasmid was called pHoxHup-NSI. To make pHoxHup-NSII, the entire cluster of T. roseopersicina genes was digested with NcoI and SpeI, and ligated into an NcoI/SpeIdigested pTRC-NSII vector.

\section{Hydrogenase activity assays}

In vitro hydrogen evolution assays were performed as described in [28] with the following modifications. Cells $(500 \mathrm{ml})$ were centrifuged, resuspended in $1 \mathrm{ml}$ sonication buffer $(10 \mathrm{mM}$ Tris$\mathrm{HCl}, \mathrm{pH}$ 7, $0.5 \mathrm{mM}$ EDTA, $1 \mathrm{mM}$ DTT), and sonicated (under aerobic conditions) two times for 2 minutes each on ice and centrifuged to remove cell debris before being used for assays. Reactions were performed under anaerobic conditions at $30^{\circ} \mathrm{C}$ as described previously using the chemical electron donor, methyl viologen [28].

\section{Protein techniques}

SDS-polyacrylamide gel electrophoresis (SDS-PAGE) was performed according to [34]. Gels were either stained with Coomassie using the SimplyBlue SafeStain reagent (Invitrogen) or transferred to nitrocellulose for Western blotting using polyclonal rabbit antibodies specific for T. roseopersicina HynL and HynS as the primary antibodies [28,34].

\section{Supporting Information}

Figure S1 Southern blot confirmation of the $S$. elongatus hoxYH mutant (PW416). After segregation on increasing antibiotic concentration, chromosomal DNA was digested with EcoRI and HindIII for Southern blotting from the following samples: Lane 1) Wild-type, 2) PW416-1, 3) PW416-2, and 4) PW416-3. A. Southern blot hybridized with a labeled PCR product amplified from hox $U$. B. Southern blot hybridized with a labeled PCR product amplified from hox $Y H$. C. Restriction map of the wild-type $S$. elongatus hox $Y H$ region. D. Restriction map of the PW416 mutant hox $\mathrm{YH}$ region.

(TIF)

Figure S2 PGR confirmation of the $S$. elongatus hoxYH mutant (PW416). After segregation on increasing antibiotic concentration, chromosomal DNA was isolated and used for PCR. The templates used in each lane are the following: Lane 1) Wild-type, 2) PW416-1, 3) PW416-2, 4) PW416-3, 5) no template, 6) pPW416 plasmid DNA, and 7) S. elongatus PCC 7942 chromosomal DNA. A. PCR products amplifying hoxH using primers Hox23 and Hox24 (Table S1). B. Diagram of primer binding sites in $S$. elongatus $\mathbf{C}$. PCR products amplifying hox $U$ through hoxW using primers Hox 16 and Hox1 7 (Table S1). D. Diagram of primer binding sites in wild-type $S$. elongatus hoxYH region. E. Diagram of primer binding sites in PW416 hox mutant.

(TIF)

Table S1 Primers used in the Supplemental Figures. (TIF)

Table S2 In vitro hydrogen evolution activity assay on wild-type and segregated hoxYH mutant strains.

(TIF)

\section{Acknowledgments}

We thank Dr. Yao Xu for kindly providing us with strains and expression vectors.

\section{Author Contributions}

Conceived and designed the experiments: PDW WAV YT JY P-CM HOS. Performed the experiments: PDW WAV YT QX. Analyzed the data: PDW WAV YT JY P-CM QX. Contributed reagents/materials/analysis tools: PDW WAV YT JY P-CM QX. Wrote the paper: PDW QX. 


\section{References}

1. Ghirardi ML, Posewitz MC, Maness PC, Dubini A, Yu JP, et al. (2007) Hydrogenases and hydrogen photoproduction in oxygenic photosynthetic organisms. Annu Rev Plant Biol 58: 71-91.

2. Tamagnini P, Leitao E, Oliveira P, Ferreira D, Pinto F, et al. (2007) Cyanobacterial hydrogenases: diversity, regulation and applications. FEMS Microbiol Rev 31: 692-720.

3. Vignais PM, Billoud B (2007) Occurrence, classification, and biological function of hydrogenases: an overview. Chem Rev 107: 4206-4272.

4. Thauer RK, Kaster AK, Goenrich M, Schick M, Hiromoto T, et al. (2010) Hydrogenases from methanogenic archaea, nickel, a novel cofactor, and $\mathrm{H}_{2}$ storage. Annu Rev Biochem 79: 507-536.

5. Appel J, Phunpruch S, Steinmuller K, Schulz R (2000) The bidirectional hydrogenase of Synechocystis sp. PCC 6803 works as an electron valve during photosynthesis. Arch Microbiol 173: 333-338.

6. Ananyev G, Carrieri D, Dismukes GC (2008) Optimizing Metabolic Capacity and Flux through Environmental Cues to Maximize Hydrogen Production by Cyanobacterium Arthrospira maxima. Appl Environ Microbiol 74: 6102-6113.

7. Armstrong FA (2004) Hydrogenases: active site puzzles and progress. Curr Opin Chem Biol 8: 133-140.

8. Cournac L, Guedeney G, Peltier G, Vignais PM (2004) Sustained Photoevolution of Molecular Hydrogen in a Mutant of Synechocystis sp. Strain PCC 6803 Deficient in the Type I NADPH-Dehydrogenase Complex. J Bacteriol 186: 1737-1746.

9. Burgdorf T, Lenz O, Buhrke T, van der Linden E, Jones AK, et al. (2005) [NiFe]-hydrogenases of Ralstonia eutropha H16: modular enzymes for oxygentolerant biological hydrogen oxidation. J Mol Microb Biotech 10: 181-196.

10. Maness PC, Smolinski S, Dillon AC, Heben MJ, Weaver PF (2002) Characterization of the Oxygen Tolerance of a Hydrogenase Linked to a Carbon Monoxide Oxidation Pathway in Rubrivivax gelatinosus. Appl Environ Microbiol 68: 2633-2636.

11. Vargas WA, Weyman PD, Tong Y, Smith HO, Xu Q (2011) A [NiFe]Hydrogenase from Alteromonas macleodii with unusual stability in the presence of oxygen and high temperature. Appl Environ Microbiol 77: 1990-1998.

12. Bock A, King PW, Blokesch M, Posewitz MC (2006) Maturation of hydrogenases. Adv Microb Physiol 51: 1-71.

13. Manyani H, Rey L, Palacios JM, Imperial J, Ruiz-Argueso T (2005) Gene Products of the hupGHIJ Operon Are Involved in Maturation of the Iron-Sulfur Subunit of the $[\mathrm{NiFe}]$ Hydrogenase from Rhizobium leguminosarum bv. viciae. J Bacteriol 187: 7018-7026.

14. Sun J, Hopkins RC, Jenney FE, Jr., McTernan PM, Adams MWW (2010) Heterologous Expression and Maturation of an NADP-Dependent [NiFe]Hydrogenase: A Key Enzyme in Biofuel Production. PLoS ONE 5: e10526.

15. Rousset M, Magro V, Forget N, Guigliarelli B, Belaich JP, et al. (1998) Heterologous Expression of the Desulfovibrio gigas [NiFe] Hydrogenase in Desulfovibrio fructosovorans MR400. J Bacteriol 180: 4982-4986.

16. Weyman PD, Vargas WA, Chuang RY, Chang Y, Smith HO, et al. (2011) Heterologous Expression of Alteromonas macleodii and Thiocapsa roseopersicina [NiFe] Hydrogenases in E. coli. Microbiology 157: 1363-1374.

17. Kovacs KL, Fodor B, Kovacs AT, Csanadi G, Maroti G, et al. (2002) Hydrogenases, accessory genes and the regulation of $[\mathrm{NiFe}]$ hydrogenase biosynthesis in Thiocapsa roseopersicina. Int J Hydrogen Energ 27: 1463-1469.

18. Colbeau A, Kovacs KL, Chabert J, Vignais PM (1994) Cloning and sequence of the structural (hupSLC) and accessory (hupDHI) genes for hydrogenase biosynthesis in Thiocapsa roseopersicina. Gene 140: 25-31.

19. Fodor B, Rakhely G, Kovacs AT, Kovacs KL (2001) Transposon mutagenesis in purple sulfur photosynthetic bacteria: identification of $h y p F$, encoding a protein capable of processing [NiFe] hydrogenases in alpha, beta, and gamma subdivisions of the proteobacteria. Appl Environ Microbiol 67: 2476-2483.

20. Xu Y, Mori T, Johnson CH (2000) Circadian clock-protein expression in cyanobacteria: rhythms and phase setting. EMBO J 19: 3349-3357.
21. Andersson CR, Tsinoremas NF, Shelton J, Lebedeva NV, Yarrow J, et al. (2000) Application of bioluminescence to the study of circadian rhythms in cyanobacteria. Method Enzymol 305: 527-542.

22. Rakhely G, Colbeau A, Garin J, Vignais PM, Kovacs KL (1998) Unusual organization of the genes coding for HydSL, the stable [NiFe] hydrogenase in the photosynthetic bacterium Thiocapsa roseopersicina BBS. J Bacteriol 180: 1460-1465.

23. Palagyi-Meszaros LS, Maroti J, Latinovics D, Balogh T, Klement E, et al. (2009) Electron-transfer subunits of the NiFe hydrogenases in Thiocapsa roseopersicina BBS. FEBS J 276: 164-174.

24. Rakhely G, Kovacs AT, Maroti G, Fodor BD, Csanadi G, et al. (2004) Cyanobacterial-Type, Heteropentameric, NAD+-Reducing NiFe Hydrogenase in the Purple Sulfur Photosynthetic Bacterium Thiocapsa roseopersicina. Appl Environ Microbiol 70: 722-728.

25. Friedrich B, Friedrich GG, Meyer M, Schlegel HG (1984) Expression of hydrogenase in Alcaligenes spp. is altered by interspecific plasmid exchange. J Bacteriol 158: 331-333.

26. Bascones E, Imperial J, Ruiz-Argueso T, Palacios JM (2000) Generation of New Hydrogen-Recycling Rhizobiaceae Strains by Introduction of a Novel hup Minitransposon. Appl Environ Microbiol 66: 4292-4299.

27. Yagi K, Min H, Urushihara M, Manabe Y, Umeda F, et al. (1986) Isolation of hydrogen-oxidation gene from Alcaligenes hydrogenophilus and its expression in Pseudomonas oxalaticus. Biochem Bioph Res Co 137: 114-119.

28. Maroti G, Tong Y, Yooseph S, Baden-Tillson H, Smith HO, et al. (2009) Discovery of a [NiFe]-hydrogenase in metagenomic Sargasso Sea DNA: Cloning and Functional Analysis in Thiocapsa roseopersicina. Appl Environ Microbiol 75: 5821-5830.

29. Ghirardi ML, Dubini A, Yu J, Maness PC (2009) Photobiological hydrogenproducing systems. Chem Soc Rev 38: 52-61.

30. Melis A, Zhang LP, Forestier M, Ghirardi ML, Seibert M (2000) Sustained photobiological hydrogen gas production upon reversible inactivation of oxygen evolution in the green alga Chlamydomonas reinhardtii. Plant Physiol 122: 127-135.

31. Clerico EM, Ditty JL, Golden SS (2007) Specialized Techniques for SiteDirected Mutagenesis in Cyanobacteria. In: Rosato E, ed. Circadian Rhythms: Methods and Protocols. Totowa: Humana Press. pp 155-171.

32. Hoffmann D, Gutekunst K, Klissenbauer M, Schulz-Friedrich R, Appel J (2006) Mutagenesis of hydrogenase accessory genes of Synechocystis sp. PCC 6803. FEBS J 273: 4516-4527.

33. Maróti G, Rákhely G, Maróti J, Dorogházi E, Klement E, et al. (2010) Specificity and selectivity of $\mathrm{HypC}$ chaperonins and endopeptidases in the molecular assembly machinery of [NiFe] hydrogenases of Thiocapsa roseopersicina. International Journal of Hydrogen Energy 35: 3358-3370.

34. Sambrook J, Russell DW (2001) Molecular Cloning: a laboratory manual, $3^{\text {rd }}$ ed. Cold Spring Harbor: Cold Spring Harbor Laboratory Press.

35. Castenholz RW (1988) Culturing Methods for Cyanobacteria. Method Enzymol 167: 68-93.

36. Boison G, Schmitz O, Mikheeva L, Shestakov S, Bothe H (1996) Cloning, molecular analysis and insertional mutagenesis of the bidirectional hydrogenase genes from the cyanobacterium Anacystis nidulans. FEBS Lett 394: 153-158.

37. Gibson DG, Young L, Chuang RY, Venter JC, Hutchison CA, et al. (2009) Enzymatic assembly of DNA molecules up to several hundred kilobases. Nat Meth 6: 343-345.

38. Ivars-Martinez E, Martin-Cuadrado AB, D'Auria G, Mira A, Ferriera S, et al (2008) Comparative genomics of two ecotypes of the marine planktonic copiotroph Alteromonas macleodii suggests alternative lifestyles associated with different kinds of particulate organic matter. ISME J 2: 1194-1212.

39. Bogorov LV (1974) The properties of Thiocapsa roseopersicina, strain BBS, isolated from an estuary of the White Sea. Mikrobiologiia 43: 326-332. 